\title{
Controlling the spiking activity in excitable membranes via poisoning
}

\author{
Gerhard Schmid, Igor Goychuk, Peter Hänggi* \\ Universität Augsburg, Institut für Physik, Theoretische Physik I, D-86135 Augsburg, Germany \\ Available online 17 July 2004
}

\begin{abstract}
The influence of intrinsic channel noise on the spontaneous spiking activity of poisoned excitable membrane patches is studied by use of a stochastic generalization of the Hodgkin-Huxley model. Internal noise stemming from the stochastic dynamics of individual ion channels is known to affect the collective properties of the whole ion channel cluster. There exists an optimal size of the membrane patch for which, solely, the internal noise causes a most regular spontaneous generation of action potentials. In addition to the variation of the size of ion channel clusters, living organisms may adopt the densities of ion channels in order to optimally regulate the spontaneous spiking activity. In our model, we selectively control via poisoning the densities of specific, active ion channels. Interestingly enough, by such poisoning of some of the potassium, or the sodium ion channels, respectively, it is possible to either increase, or decrease the regularity of the spike train.
\end{abstract}

(c) 2004 Elsevier B.V. All rights reserved.

PACS: 05.40.-a; 87.10.+e; 87.16.-b

Keywords: Excitable membranes; Ion channels; Channel noise

\section{Introduction}

Following the study of Hodgkin and Huxley [1], most of the models of axons have treated the generation and propagation of action potentials using deterministic differential equations. But since Lecar and Nossal [2], it is taken for granted that the randomness of the ion channel gating causes threshold fluctuations in neurons. Therefore, channel noise which stems from the stochastic nature of the ion channel dynamics

\footnotetext{
* Corresponding author. Tel.: +49-821-598-3250; fax: +49-821-598-3222.

E-mail addresses: gerhard.schmid@physik.uni-augsburg.de (G. Schmid), peter.hanggi@physik.uni-augsburg.de (P. Hänggi).
} 
cannot be disregarded [3]; it impacts, e.g., features such as the threshold to spiking, the spiking rate itself [4-7] and the efficiency for synchronization [8]. The strength of the channel noise is mainly determined by the number of ion channels participating in the generation of action potentials. Interestingly, there are optimal patch sizes for which the spike production is mostly regular [6,7]. The objective addressed with this work is how the regularity of the spiking can possibly be controlled for a given patch size of excitable ion channels. There are toxins like tetraethylammonium (TEA) and tetrodotoxin (TTX), respectively, which allow reducing the number of working potassium and/or sodium ion channels [9]. Thereby the level of the intrinsic noise becomes a priori controllable.

\section{A stochastic Hodgkin-Huxley model}

Fox and Lu developed a stochastic extension [4] of the Hodgkin-Huxley model [1] which accounts for channel noise. The origin of the channel noise [3] is basically due to fluctuations of the number of open ion channels around the corresponding mean values. According to the Hodgkin-Huxley model, the dynamics of the membrane potential $V$ is given by

$$
C \frac{\mathrm{d}}{\mathrm{d} t} V+G_{\mathrm{K}}(n)\left(V-E_{\mathrm{K}}\right)+G_{\mathrm{Na}}(m, h)\left(V-E_{\mathrm{Na}}\right)+G_{\mathrm{L}}\left(V-E_{\mathrm{L}}\right)=0,
$$

if an external stimulus is absent. In Eq. (1), $C=1 \mu \mathrm{F} / \mathrm{cm}^{2}$ is the capacity of the cell membrane. Furthermore, $E_{\mathrm{Na}}=50 \mathrm{mV}, E_{\mathrm{K}}=-77 \mathrm{mV}$ and $E_{\mathrm{L}}=-54.4 \mathrm{mV}$ are the reversal potentials for the potassium, sodium and leakage currents, correspondingly. While the leakage conductance is assumed to be constant, $G_{\mathrm{L}}=0.3 \mathrm{mS} / \mathrm{cm}^{2}$, the potassium and sodium conductances read:

$$
G_{\mathrm{K}}(n)=g_{\mathrm{K}}^{\max } x_{\mathrm{K}} n^{4}, \quad G_{\mathrm{Na}}(m, h)=g_{\mathrm{Na}}^{\max } x_{\mathrm{Na}} m^{3} h,
$$

where $g_{\mathrm{K}}^{\max }=36 \mathrm{mS} / \mathrm{cm}^{2}$ and $g_{\mathrm{Na}}^{\max }=120 \mathrm{mS} / \mathrm{cm}^{2}$ denote the maximal conductances (when all the channels are open). In Eq. (2), we introduce the factors $x_{\mathrm{K}}$ and $x_{\mathrm{Na}}$ which correspond to the fraction of working, i.e., non-blocked ion channels, to the overall number of potassium, $N_{\mathrm{K}}$, or sodium, $N_{\mathrm{Na}}$, ion channels, correspondingly. These factors are confined to the unit interval. Experimentally, they can be controlled by adding cell toxins like TEA and/or TTX which completely block and disable potassium or sodium ion channels, respectively [9].

While the gating variables $n, m$ and $h$ describe the mean ratios of the open gates of the working channels, the factors $n^{4}$ and $m^{3} h$ are the mean portions of the open ion channels within a membrane patch. This follows from the fact that the gating dynamics of each ion channel is assumed to be governed by four independent gates, each of which could switch between an open and a closed conformation. The voltage-dependent opening and closing rates $\alpha_{x}(V)$ and $\beta_{x}(V)(x=m, h, n)$, read [6,7]:

$$
\alpha_{m}(V)=\frac{0.1(V+40)}{1-\exp [-(V+40) / 10]},
$$




$$
\begin{aligned}
& \beta_{m}(V)=4 \exp [-(V+65) / 18], \\
& \alpha_{h}(V)=0.07 \exp [-(V+65) / 20], \\
& \beta_{h}(V)=\{1+\exp [-(V+35) / 10]\}^{-1}, \\
& \alpha_{n}(V)=\frac{0.01(V+55)}{1-\exp [-(V+55) / 10]}, \\
& \beta_{n}(V)=0.125 \exp [-(V+65) / 80] .
\end{aligned}
$$

The gating variables obey the following Langevin equations [4]:

$$
\frac{\mathrm{d}}{\mathrm{dt}} x=\alpha_{x}(V)(1-x)-\beta_{x}(V) x+\xi_{x}(t), \quad x=m, h, n,
$$

with independent Gaussian white noise sources $\xi_{x}(t)$ of vanishing mean. The noise correlations assume the following form for an excitable membrane patch with $N_{\mathrm{Na}}$ sodium and $N_{\mathrm{K}}$ potassium ion channels:

$$
\begin{aligned}
\left\langle\xi_{m}(t) \xi_{m}\left(t^{\prime}\right)\right\rangle & =\frac{2}{N_{\mathrm{Na}} x_{\mathrm{Na}}} \frac{\alpha_{m} \beta_{m}}{\left(\alpha_{m}+\beta_{m}\right)} \delta\left(t-t^{\prime}\right), \\
\left\langle\xi_{h}(t) \xi_{h}\left(t^{\prime}\right)\right\rangle & =\frac{2}{N_{\mathrm{Na}} x_{\mathrm{Na}}} \frac{\alpha_{h} \beta_{h}}{\left(\alpha_{h}+\beta_{h}\right)} \delta\left(t-t^{\prime}\right), \\
\left\langle\xi_{n}(t) \xi_{n}\left(t^{\prime}\right)\right\rangle & =\frac{2}{N_{\mathrm{K}} x_{\mathrm{K}}} \frac{\alpha_{n} \beta_{n}}{\left(\alpha_{n}+\beta_{n}\right)} \delta\left(t-t^{\prime}\right) .
\end{aligned}
$$

The overall numbers of involved potassium and sodium ion channels are re-scaled by $x_{\mathrm{Na}}$ and $x_{\mathrm{K}}$, respectively, in order to disregard the blocked channels which do not contribute to the channel noise. With the assumption of homogeneous ion channel densities, $\rho_{\mathrm{Na}}=60 \mu \mathrm{m}^{-2}$ and $\rho_{\mathrm{K}}=18 \mu \mathrm{m}^{-2}$, the ion channel numbers are given by: $N_{\mathrm{Na}}=\rho_{\mathrm{Na}} S, N_{\mathrm{K}}=\rho_{\mathrm{K}} S$, with $S$ being the size of the membrane patch. The number of working ion channels scaled with the size of the excitable membrane patch $S$ determines the strength of the fluctuations. With decreasing patch size, i.e., decreasing number of ion channels, the noise level caused by fluctuations of the number of open ion channels increases, cf. Eq. (5).

The numerical integration of this stochastic generalized Hodgkin-Huxley model, cf. Eqs. (1)-(5), is carried out by the standard Euler algorithm with a step size of $1 \mu$ s. The Gaussian random numbers are generated by the "Numerical Recipes" routine ran2 using the Box-Muller algorithm [10]. To ensure the confinement of the gating variables between 0 (all gates are closed) and 1 (all gates are open), we implemented numerically the reflecting boundaries at 0 and 1 . The occurrences of action potentials are determined by upward crossings of the membrane potential $V$ of a certain detection threshold. Due to the very steep increase of membrane potential at firing, the actual choice of the detection threshold does not affect the results. In our simulations, the spontaneous spikes are determined by upward crossings at zero threshold voltage. The occurrences 
of action spikes $t_{i}$, where $i=1, \ldots, N$ form a point process with certain statistical properties which are discussed next.

\section{The mean interspike interval}

While in the original deterministic Hodgkin-Huxley model, the action potentials occur only for a certain external current stimulus, the intrinsic channel noise initiates spontaneous spikes $[3,5,11-13]$. The mean interspike interval, i.e.,

$$
\langle T\rangle=\frac{1}{N} \sum_{i=1}^{N}\left(t_{i}-t_{i-1}\right),
$$

with $t_{0}=0$, becomes a function of patch size $S$. The inverse mean interspike interval defines the spiking rate. With increasing noise level or decreasing patch sizes $S$, the spike production increases and thus the mean interspike interval $\langle T\rangle$ decreases and can approach the refractory time [6,7].

In Fig. 1, we depict the mean interspike interval $\langle T\rangle$ against the fractions $x_{\mathrm{Na}}$ and $x_{\mathrm{K}}$ of working sodium channels or potassium ion channels, respectively, for different patch sizes $S$. A reduction of the number of working sodium ion channels, i.e., smaller $x_{\mathrm{Na}}$, increases the strength of channel noise which is caused by the stochastic behavior of the sodium ion channels [5]. Because the channel noise, which is induced by the sodium channels, is mainly responsible for the initiation of action potentials from the rest potential, one might expect that a reduction of the number of sodium ion channels could then lead to more spikes. On the contrary, however, a reduction of the number of working sodium channels causes a diminishment of the sodium conductance, cf. Eq. (2); it is the latter effect that dominates and consequently causes an increase of the mean interspike interval, cf. Fig. 1(a).

The reduction of potassium conductance by poisoning the potassium channels changes dramatically the dynamics of the original Hodgkin-Huxley model which ignores the
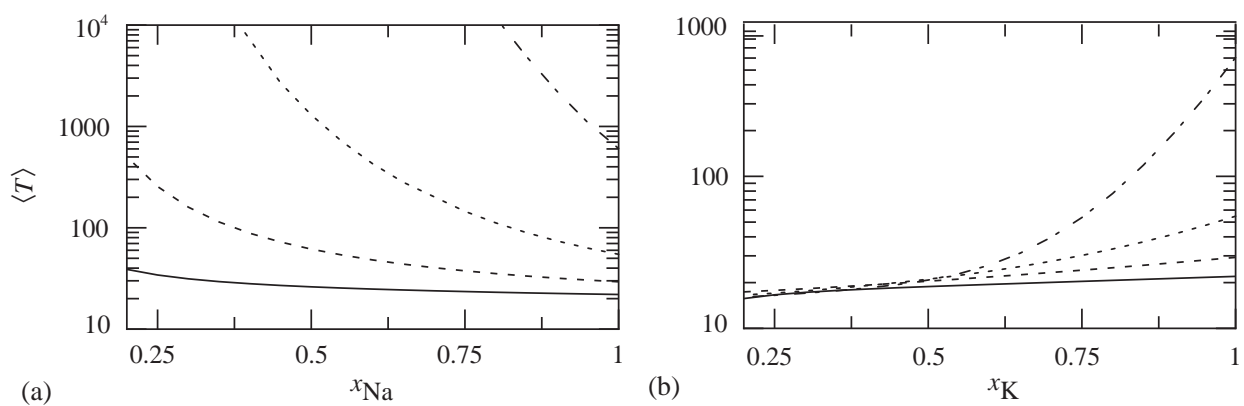

Fig. 1. Mean interspike interval for poisoning. The dependence of the mean time between two subsequent, spontaneous action potentials versus: (a) the ratio of intact sodium channels and (b) the fraction of active potassium ion channels is shown for four different patch sizes: $S=1 \mu \mathrm{m}^{2}$ (solid line), $S=4 \mu \mathrm{m}^{2}$ (dashed line), $S=16 \mu \mathrm{m}^{2}$ (dotted line), and $S=64 \mu \mathrm{m}^{2}$ (dash-dotted line). 
impact of channel noise. With decreasing potassium conductance, a sub-critical Hopfbifurcation for $x_{\mathrm{K}}=0.5490$ takes place in the deterministic model. By the poisoning of potassium channels, the system thus enters the region where a stable spiking solution exists within a purely deterministic description. The frequency of spiking is determined by the refractory period of action potentials. Hence, the mean interspike interval $\langle T\rangle$ decreases with a reduction of working potassium channels $x_{\mathrm{K}}$. This fact is confirmed with Fig. 1(b).

\section{Controlling the coherence of poisoned spiking activity}

We next address the regularity of spontaneous action potentials. A proper measure is the coefficient of variation, $C V$, a measure of coherence, which is given as the ratio of standard deviation to the mean value

$$
C V=\frac{\sqrt{\left\langle T^{2}\right\rangle-\langle T\rangle^{2}}}{\langle T\rangle},
$$

where $\left\langle T^{2}\right\rangle:=1 / N \sum\left(t_{i}-t_{i-1}\right)^{2}$ is the mean-squared interspike interval. For a fully disordered point process (the case of Poisson process), the coefficient of variation $C V$ assumes the value $C V=1$, while for more ordered processes, it assumes smaller values, and for a deterministic signal it vanishes altogether. In previous studies, it has been demonstrated that $C V$ exhibits a distinct minimum for an optimal patch size $S \approx 1 \mu \mathrm{m}^{2}$ at which the spiking is most regular [6,7]. This phenomenon has been termed as intrinsic coherence resonance.

In Fig. 2, the coefficient of variation is plotted against $x_{\mathrm{Na}}$ (a) and $x_{\mathrm{K}}$ (b). Any addition of TTX which blocks sodium ion channels leads to an increase of the $C V$, cf. Fig. 2(a). In contrast, toxins which disable potassium channels yield a rise of the regularity beyond the value reached by optimizing the patch size only, cf. Ref. [6]. This result is due to the existence of a regime of potassium conductance level possessing a stable oscillatory spiking solution in the deterministic model. The channel noise then

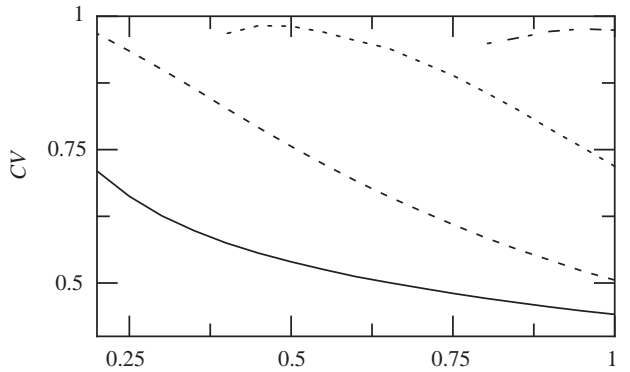

(a)

$$
x_{\mathrm{Na}}
$$

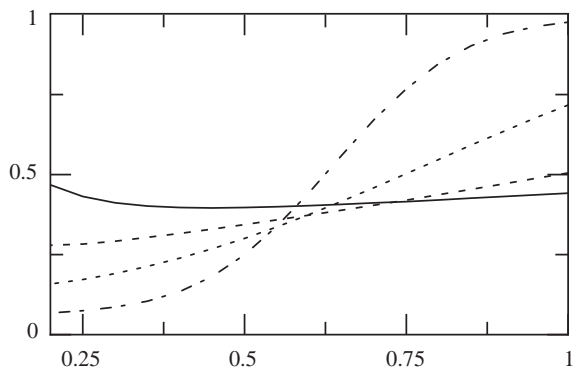

(b)

Fig. 2. (a), (b) Same as in Fig. 1, but for the coefficient of variation $C V$, cf. Eq. (7). By addition of TEA or TTX, the regularity of the spiky output could be both increased and decreased. 
disturbs the regular spiking at a fixed frequency, yielding the observed behavior for the $C V$.

\section{Conclusions}

Our study of the stochastically generalized Hodgkin-Huxley model reveals the possibility to manipulate a priori the response of a spiking membrane patch by adding toxins, which selectively block the ion channels. For example, by a fine-tuned addition of tetrodotoxin, a certain portion of sodium ion channels could be experimentally disabled. This in turn results in a reduction of the spontaneous action potentials and yields a more irregular production of spikes. On the contrary, the addition of tetraethylammonium can be used in order to block potassium ion channels. This causes an unexpected increase of the spiking activity (i.e., a decrease of the mean interspike interval) and yields a more regular spontaneous spiking coherence. These characteristic features are expected to impact the behavior of biological stochastic resonance $[14,15]$ in these systems, see e.g. in Ref. [6].

\section{Acknowledgements}

This work has been supported by the Deutsche Forschungsgemeinschaft via the Sonderforschungsbereich SFB-486, Project A10.

\section{References}

[1] A.L. Hodgkin, A.F. Huxley, J. Physiol. (London) 117 (1952) 500.

[2] H. Lecar, R. Nossal, Biophys. J. 11 (1971) 1048.

[3] J.A. White, J.T. Rubinstein, A.R. Kay, Trends Neurosci. 23 (2000) 131

[4] R.F. Fox, Y. Lu, Phys. Rev. E 49 (1994) 3421.

[5] C.C. Chow, J.A. White, Biophys. J. 71 (1996) 3013.

[6] G. Schmid, I. Goychuk, P. Hänggi, Europhys. Lett. 56 (2001) 22.

[7] P. Jung, J.W. Shuai, Europhys. Lett. 56 (2001) 29.

[8] G. Schmid, I. Goychuk, P. Hänggi, Physica A 325 (2003) 165.

[9] B. Hille, Ionic Channels of Excitable Membranes, 3rd Edition, Sinauer Associates, Sunderland, MA, 2001.

[10] W.H. Press, S.A. Teukolsky, W.T. Vetterling, B.P. Flannery, Numerical Recipes in C, 2nd Edition, Cambridge University Press, Cambridge, 1992.

[11] J.R. Clay, L.J. DeFelice, Biophys. J. 42 (1983) 151.

[12] A.F. Strassberg, L.J. DeFelice, Neural Comput. 5 (1993) 843.

[13] L.J. DeFelice, A. Isaac, J. Stat. Phys. 70 (1993) 339.

[14] L. Gammaitoni, P. Hänggi, P. Jung, F. Marchesoni, Rev. Mod. Phys. 70 (1998) 223.

[15] P. Hänggi, Chem. Phys. Chem. 3 (2002) 285. 